\title{
A Molecular Diagnostic Test for Persistent Müllerian Duct Syndrome in Miniature Schnauzer Dogs
}

\author{
S. Pujar V.N. Meyers-Wallen \\ Baker Institute for Animal Health, Cornell University, College of Veterinary Medicine, Ithaca, N.Y., USA
}

\section{Key Words}

DNA test $\cdot$ Dog $\cdot$ Miniature schnauzer $\cdot$ Persistent Müllerian duct syndrome

\begin{abstract}
In persistent Müllerian duct syndrome (PMDS), Müllerian ducts fail to regress in males during sexual differentiation. In the canine miniature schnauzer model, PMDS is caused by a $C$ to $T$ transition in exon 3 of the Müllerian inhibiting substance type II receptor (MISRII), which introduces a Ddel restriction site. Here we report a molecular diagnostic test for PMDS in the miniature schnauzer to identify affected dogs and carriers. As our test results suggest that the mutation is identical by descent in affected dogs of this breed, the test could be used to eliminate this mutation from the miniature schnauzer breed worldwide. Copyright $\odot 2009$ S. Karger AG, Basel
\end{abstract}

Persistent Müllerian duct syndrome (PMDS) is a rare form of male pseudohermaphroditism wherein Müllerian duct derivatives are retained in otherwise normal males. Canine PMDS has been reported in the miniature schnauzer in North America [Brown et al., 1976; Marshall et al., 1982; Meyers-Wallen et al., 1989], Europe [Vegter et al., 2008] and Asia [Matsuu et al., 2009]. Affected miniature schnauzers are $78, \mathrm{XY}$ males that have bilateral testes and normal androgen-dependent masculinization of the internal and external genitalia. However, all Müllerian duct derivatives, bilateral oviducts, a complete uterus with cervix and the cranial portion of the vagina, are also present. Approximately $50 \%$ of affected dogs have normally descended testes and are fertile [Meyers-Wallen et al., 1989]. However, the remaining 50\% have unilateral or bilateral cryptorchidism, and as a result can be infertile and develop testicular tumors [Brown et al., 1976; Vegter et al., 2008; Matsuu et al., 2009]. Treatment involves surgical procedures (orchidectomy and hysterectomy) and significant expenses for dog owners. Prevention is limited to the elimination of affected dogs and carriers from the breeding population. In the miniature schnauzer, PMDS is inherited as an autosomal recessive trait with expression limited to homozygous males [Meyers-Wallen et al., 1989]. Both males and females can be carriers. However, affected dogs cannot be identified by physical examination alone, particularly if they are not cryptorchid. Similarly, carriers cannot be detected, as they are reproductively normal males or females. Diagnosis and prevention could be facilitated by a DNA-based test to identify all affected dogs and carriers.

The causative molecular defect for PMDS in the miniature schnauzer is a $\mathrm{C}$ to $\mathrm{T}$ transition (C241T) in exon 3 of the Müllerian inhibiting substance type II receptor gene, MISRII (AMHR2) [Wu et al., 2009], which was identical by descent in the model pedigree. The purpose

\section{KARGER}

(C) 2009 S. Karger AG, Basel

Fax +4161306 1234

E-Mail karger@karger.ch

www.karger.com
Accessible online at:

www.karger.com/sxd
Vicki N. Meyers-Wallen

Baker Institute for Animal Health

Cornell University, College of Veterinary Medicine

Ithaca, NY 14853 (USA)

Tel. +1 607256 5683, Fax +1 607256 5608, E-Mail vnm1@ cornell.edu 


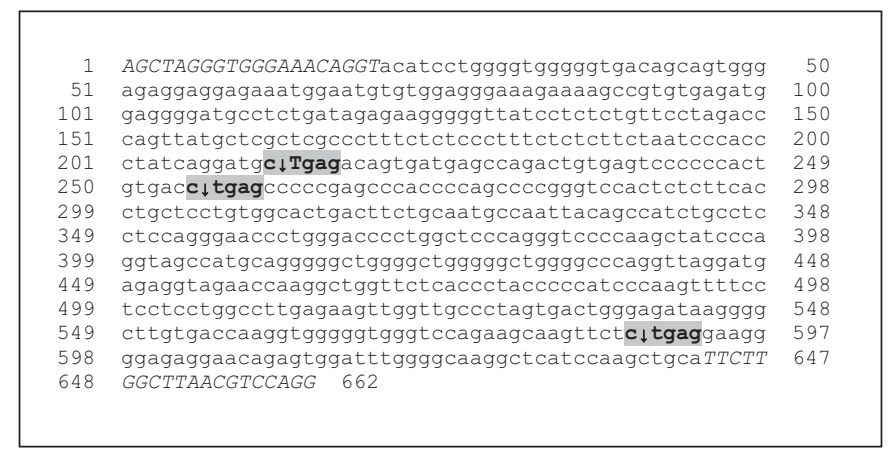

Fig. 1. DdeI restriction sites (shaded) within exon 3 of MISRII in the 662-bp PCR product from a PMDS affected dog. The C241T mutation ( $\mathrm{T}$ ) introduces a new DdeI restriction site. The DNA cleavage sites are indicated by arrows. Forward and reverse PCR primers are italicized and capitalized.

of this study was to design a molecular test based on this mutation, which introduces a DdeI restriction enzyme site. A second purpose was to test the hypothesis that the causative mutation for PMDS is identical by descent in the miniature schnauzer breed.

\section{Materials and Methods}

\section{Animals}

A total of 8 dogs were tested, including heterozygous carriers (A139 and A185) and affected dogs (A291 and A141) from the PMDS model pedigree, and 2 normal beagle female controls (A66 and A1013), as previously reported [Wu et al., 2009]. In addition, 2 affected miniature schnauzers unrelated to the model pedigree (PM4808 and PM4913) were included to test the hypothesis that the mutation is identical by descent in this breed.

\section{DNA Extraction}

Genomic DNA from each dog was extracted from stored whole blood samples by standard phenol and chloroform extraction with ethanol precipitation [Sambrook et al., 1989], then quantified by spectrophotometry (Nanodrop spectrophotometer, Wilmington, DE).

\section{Primers and PCR}

Primers were designed using Primer3 (http://frodo.wi.mit. edu/primer3/) and synthesized by Integrated DNA Technologies (IDT, Coralville, Iowa, USA). The primers, forward 5'-AGCTAGGGTGGGAAACAGGT-3' and reverse 5'-CCTGGACGTTAAGCCAAGAA-3', amplify a 662-bp product within exon 3 of canine MISRII that includes the C241T mutation. PCR reactions and thermal cycling conditions were as described in $\mathrm{Wu}$ et al. [2009] with the exception that annealing temperature was lowered stepwise as follows: $59^{\circ} \mathrm{C}$ ( 4 cycles), $58^{\circ} \mathrm{C}$ ( 4 cycles), $57^{\circ} \mathrm{C}$ ( 4 cycles) and finally $56^{\circ} \mathrm{C}$ ( 23 cycles). PCR products were separated by electrophoresis in a $2.5 \%$ agarose gel stained with ethidium bromide.

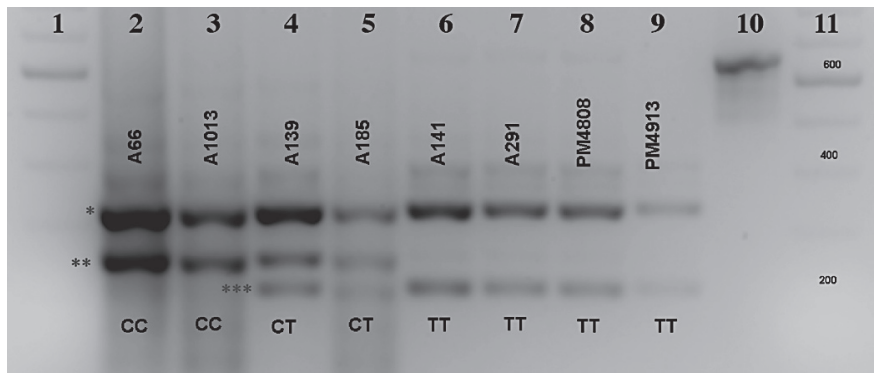

Fig. 2. Separation of $D d e I$ digestion products (2.5\% agarose gel, reverse contrast). Lanes 1 and 11: 100-bp ladder; lanes 2 and 3: normal dogs; lanes 4 and 5: heterozygous carriers; lanes 6-9: PMDS-affected dogs; lane 10: undigested 662-bp PCR product. The 3 diagnostic bands are indicated by asterisks: $333 \mathrm{bp}\left(^{*}\right), 255$ bp $\left({ }^{* *}\right)$ and 212 bp $\left({ }^{* *}\right)$. Dog identification numbers are above the bands and genotypes at the C241T site are below the bands.

The 662-bp band was excised from the gel and purified using the QiaQuick Gel Extraction kit (Qiagen, Valencia, Calif., USA).

\section{Restriction Enzyme Digestion}

A restriction enzyme search program (http://tools.neb.com/ NEBcutter2/index.php) was used to identify restriction sites within the 662-bp PCR product. Restriction digestion was carried out in a reaction mix containing $0.6 \mu \mathrm{l}$ of $100 \mathrm{mM}$ spermidine, $4 \mu \mathrm{l}$ of $10 \times$ NEBuffer 3 (New England Biolabs-NEB, Ipswich, Mass., USA), 2 units of DdeI restriction enzyme, 10-20 $\mu \mathrm{l}(2 \mu \mathrm{g})$ of the purified PCR band and MilliQ water sufficient for $40 \mu$ l total reaction volume. Reactions were incubated at $37^{\circ} \mathrm{C}$ for $4 \mathrm{~h}$. Digestion products were separated by electrophoresis and photographed.

\section{Sequencing}

To confirm genotypes identified by restriction digestion, the 662-bp PCR product for each dog was also sequenced at the Cornell Life Science Core Laboratories using the BigDye Terminator kit (Applied Biosystems, Foster City, Calif., USA). Sequences were aligned for comparison using ClustalW (http://www.ebi.ac.uk/ Tools/clustalw/index.html).

\section{Results}

Digestion of the 662-bp PCR product with DdeI (fig. 1) yields the following products: normal (CC): $255 \mathrm{bp}, 333$ bp and $74 \mathrm{bp}$; heterozygote (CT): $255 \mathrm{bp}, 212 \mathrm{bp}, 43 \mathrm{bp}$, $333 \mathrm{bp}$ and $74 \mathrm{bp}$; affected (TT): $212 \mathrm{bp}, 43 \mathrm{bp}, 333 \mathrm{bp}$ and $74 \mathrm{bp}$. The 3 products that differentiate the genotypes (212 bp, $255 \mathrm{bp}$ and $333 \mathrm{bp}$ ) are clearly visible after agarose gel electrophoresis (fig. 2). The 43-bp and 74-bp products are not visible under these conditions. Sequence alignment confirmed all genotypes (online suppl. fig. 1, see www.karger.com/doi/10.1159/000273264). 


\section{Discussion}

Test results for all dogs were concordant with their disease status. This included the 2 affected miniature schnauzers (PM4808 and PM4913) that are unrelated to the PMDS model pedigree in which the mutation was first identified [Wu et al., 2009]. As the causative mutation should be identical by descent in purebred miniature schnauzers, and the C241T mutation is the only mutation that has been identified in affected dogs of this breed, this mutation is the only confirmed cause of PMDS in purebred miniature schnauzers. Therefore this test could be used to eliminate the mutation from this breed throughout the world, improving the health of the breed as a whole. The 2 main steps involved in the test, PCR amplification and gel electrophoresis, are amenable to automation, making rapid, high-throughput diagnosis feasible.

Since cryptorchidism, which occurs in approximately $50 \%$ of PMDS affected miniature schnauzers, renders them sterile or subfertile, they are of little breeding value to owners and breeders. Cryptorchid dogs are also susceptible to Sertoli cell tumors [Brown et al., 1976; Marshall et al., 1982] and pyometra [Marshall et al., 1982], which encumbers owners with additional treatment expenses. Furthermore, the test reported here is the only noninvasive method to definitively diagnose PMDS dogs that are not cryptorchid, which constitute approximately half of the affected miniature schnauzers. They have no external signs of the disorder and can be fertile. Left undetected, a fertile PMDS male that is a popular sire could rapidly increase the frequency of the mutant allele in the breed worldwide, given the present ability to cryopreserve and distribute canine semen. Therefore it is essential to reduce the frequency of the mutant allele in the population, and to eventually eliminate it from the miniature schnauzer breed.

In summary, the test reported here is a valuable selection tool to positively identify affected dogs before they are bred and remove them from the breeding pool. It can also identify male and female carriers, allowing breeders to avoid breeding carriers together, and prevent the production of affected dogs. After reducing the mutant allele frequency, it should be possible to eliminate this mutation entirely from the miniature schnauzer breed to improve the health of the breed as a whole.

\section{Acknowledgements}

The authors thank Shana Mintz for technical assistance and Anita Hesser for manuscript preparation. A portion of this study was supported by NIH R01HD19393 (V.M.-W.).

\section{References}

Brown TT, Burek JD, McEntee K: Male pseudohermaphroditism, cryptorchidism and Sertoli cell neoplasia in three miniature schnauzers. J Am Vet Med Assoc 169:821-825 (1976).

Marshall LS, Oehlert ML, Haskins ME, Selden JR, Patterson DF: Persistent Müllerian duct syndrome in miniature schnauzers. J Am Vet Med Assoc 81:798-801 (1982).
Matsuu A, Hashizume T, Kanda K, Nagano M, Sugiyama A, et al: A case of persistent Müllerian duct syndrome with Sertoli cell tumor and hydrometra in a dog. J Vet Med Sci 71: 379-381 (2009).

Meyers-Wallen VN, Donahoe PK, Ueno S, Manganaro TF, Patterson DF: Müllerian inhibiting substance is present in testes of dogs with persistent Müllerian duct syndrome. Biol Reprod 41:881-888 (1989).

Sambrook J, Fritsch EF, Maniatis T: Molecular Cloning: A Laboratory Manual, 2nd ed., pp E3-E15 (Cold Spring Harbor Laboratory Press, Cold Spring Harbor 1989).
Vegter AR, Kooistra HS, van Sluijs FJ, van Bruggen LWL, Izjer J, et al: Persistent Müllerian Duct Syndrome in a miniature schnauzer dog with signs of feminization and a Sertoli cell tumor. Reprod Domest Anim, doi:10.1111/j.1439-0531.2008.01223.x(2008).

Wu X, Wan S, Pujar S, Haskins ME, Schlafer DH, et al: A single base pair mutation encoding a premature stop codon in the MIS type II receptor is responsible for canine persistent Müllerian duct syndrome. J Androl 30:4656 (2009). 